\title{
RESPONSABILIDADE SOCIAL CORPORATIVA E DESEMPENHO FINANCEIRO: UMA REVISÃO SISTEMÁTICA DA LITERATURA INTERNACIONAL
}

Fabio Emanuel Farago ${ }^{1}$

Guilherme Primo Matias ${ }^{2}$

${ }^{1}$ Departamento de Administração Geral e Aplicada / PPGADM - Escola de Administração / Universidade Federal do Paraná

${ }^{2}$ Departamento de Administração Geral e Aplicada / Escola de Administração - PPGADM / Universidade Federal do Paraná 


\section{RESPONSABILIDADE SOCIAL CORPORATIVA E DESEMPENHO FINANCEIRO: UMA REVISÃO SISTEMÁTICA DA LITERATURA INTERNACIONAL.}

Resumo: Neste trabalho discute-se a relação entre a responsabilidade social corporativa (RSC) e o desempenho financeiro (DF) por meio de uma revisão sistemática da literatura internacional. A relação entre a RSC e o DF tem sido amplamente debatida, entretanto, a literatura se divide entre trabalhos que sugerem uma relação positiva entre a RSC e o DF e aqueles que afirmam uma relação negativa. A metodologia utilizada foi uma revisão sistemática em duas bases de dados internacionais: Scopus e Web of Science. O período da pesquisa foi de 2007 a 2017. Após a aplicação de filtros, foram selecionados para análise 20 artigos. Entre as principais contribuições, se destacou que $95 \%$ dos artigos são quantitativos, $75 \%$ afirmam uma relação positiva entre RSC e DF e $25 \%$ uma relação negativa. Foi observado também uma limitação metodológica na literatura, que se utiliza principalmente de modelos estatísticos tradicionais.

Palavras-chave: RSC. Desempenho social. Revisão de literatura.

\section{Introdução}

As empresas, na definição dos planos de gestão, podem se engajar em diversos tipos de ações estratégicas visando aprimorar sua posição competitiva, entre essas, a responsabilidade social corporativa (RSC) tem obtido destaque (Li, Zhou \& Shao, 2009). A RSC tem obtido cada vez maior ênfase na medida em que os empresários a identificam como uma oportunidade de obter resultados positivos ao investir em questões sociais, ambientais e éticas. Entretanto, uma questão que surge é será que as estratégias de investimentos em RSC são lucrativas?

As organizações cada vez têm investido mais recursos em áreas sociais. O interesse das empresas na RSC decorre da expectativa de melhorar o relacionamento e a transparência com os stakeholders (Baraibar-Diez \& Sotorrío, 2018). A RSC pode auxiliar uma empresa a construir sua reputação e obter maior suporte na comunidade a que está inserida, assim, melhorando seu desempenho financeiro (DF) (Kim, Kim \& Qian, 2015). Deste modo, parece existir um relacionamento positivo entre a RSC e o DF.

Entretanto, a literatura sobre RSC tem se tornado cada vez mais complexa e multifacetada (Jamali \& Karam, 2016). Os efeitos da RSC no DF têm sido investigados por
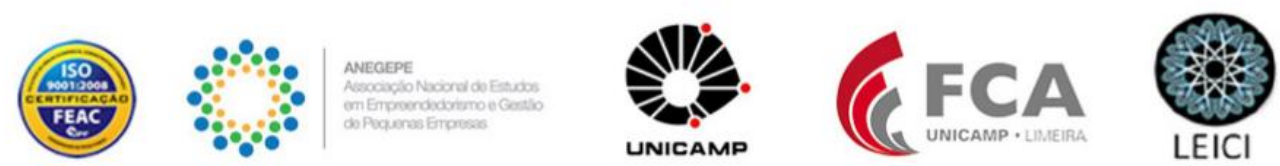
décadas com resultados mistos, deste modo, este debate ainda está por ser solucionado. Isso se deve em parte devido a um relacionamento direto entre as variáveis ter se mostrado complexo (Hull \& Rothenberg, 2008).

A relação entre a responsabilidade social de uma firma e seu desempenho financeiro tem sido debatida na literatura desde a década de 60. Entretanto, os pesquisadores não chegaram a um consenso real de como se dá o relacionamento entre essas duas variáveis (Cochran \& Wood, 1984). Posto isso, a literatura e os debates sobre os efeitos da RSC e DF se encontram divididos.

Os trabalhos que sugerem efeitos negativos entre a RSC e o DF argumentam que as firmas na tentativa de aprimorar seu desempenho social, retiram recursos e esforços de áreas chaves do negócio, ocasionando redução do lucro. Por outro lado, as pesquisas que suportam um efeito positivo entre a RSC e o DF tem afirmado que a RSC auxilia na diversificação, na expansão das oportunidades de mercado, no relacionamento com stakeholders e na retenção de mão de obra qualificada (Hull \& Rothenberg, 2008).

A existência ou não dessa relação é importante para a administração estratégica, pois se as ações classificadas como socialmente responsáveis forem negativamente correlacionadas com o desempenho financeiro, então é aconselhável que os empresários tomem cuidado nessa área. Por outro lado, se uma relação positiva existir, então os empreendedores podem ser encorajados a perseguir as atividades de responsabilidade social (Cochran \& Wood, 1984).

A tomada de decisões estratégicas e de alocação de recursos escassos sempre foi complexa, porém atualmente, isso vem se amplificando ainda mais. As empresas são cobradas não apenas em termos de seus resultados financeiros, mas também nas formas como as empresas reagem a uma série de expectativas sociais que são depositadas sobre ela (Waddock \& Graves, 1997). Por este motivo, empresas que deixem a RSC ao descaso podem ter seu relacionamento com os stakeholders afetado.

Deste modo, o relacionamento entre a RSC e o desempenho das firmas ainda se configura como um campo fragmentado. A inabilidade de se atingir um consenso tem levado a inúmeros debates. Posto isso, se destaca a importância de analisar o que a literatura atual tem apontado no relacionamento entre os conceitos. Foi observado por Jamali e Karam (2016) que apesar do crescente interesse na RSC, sobretudo em países em desenvolvimento, ainda existe uma lacuna de revisões sistemáticas dessa literatura.

Este artigo tem por objetivo contribuir com essa discussão ao elaborar uma revisão sistemática de literatura a respeito da interação entre a responsabilidade social corporativa e o desempenho financeiro. Para tanto, foram consultadas as bases de dados internacionais Scopus e Web of Science, no período de 2007 a 2017, para verificar o estado da arte da relação entre a RSC e o DF.

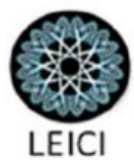




\section{Referencial Teórico}

A literatura sobre responsabilidade social corporativa (RSC), é parte integrante do desenvolvimento teórico acerca dos negócios sociais. Isto se deve em função de que os negócios sociais, conforme atenta Barki (2015), surgem como um afronte as lacunas sociais criadas pelo sistema capitalista. Os negócios sociais se caracterizam por gerar valor intrinsecamente econômico e social e consequentemente se tornam uma alternativa para combater o alto déficit social existente principalmente nos países em desenvolvimento.

Foi observado por Teodósio e Comini (2012), que no epilogo da década de 1990 houve um crescimento nos movimentos filantrópicos em diversos setores das sociedades. Entretanto, foi a partir do estudo de Prahalad e Hart (2002) e Prahalad (2005), que se atentou para a valia de se desenvolver negócios, sobretudo na base da pirâmide social. Posteriormente, Thornton e Ocasio (2008), Batilana e Dorado (2010) e Batilana e Lee (2014) abordaram as organizações híbridas, ou seja, que compartilham múltiplas lógicas (como a social e a de mercado), e a literatura acerca dos negócios sociais começou a ganhar maior notoriedade.

Diante desta tênue entre a busca da criação de valor econômico e social, Chaves, Rocha, Reuther e Galhanone (2017), acenam para o importante papel que as organizações têm na mitigação de problemas sociais e ambientais ao oferecer produtos e serviços à base da pirâmide social e expõem a forma como tais organizações utilizam-se do know-how em marketing para alcançar os resultados desejados. Contudo, ressalta-se que estes acometimentos sociais estão diretamente ligados aos valores e estratégias das empresas, ou seja, a ideia de transformação social está relacionada ao seu core business.

A literatura acerca dos negócios sociais possui três perspectivas específicas de como tal modelo negócio pode ser estruturado. A primeira delas é a norte-americana, que corresponde a empresas com de responsabilidade social corporativa; a segunda é a vertente europeia, que se refere a cooperativas capazes de auxiliar empreendedores que sozinhos não conseguiriam desenvolver seu plano de negócio; e a última, é a perspectiva dos países emergentes, que enfatiza os negócios inclusivos que atingem e/ou nascem na base da pirâmide social (Comini, Barki \& Aguiar, 2012). As três perspectivas podem ser observadas na Figura 1.

Figura 01: Perspectivas dos negócios sociais.

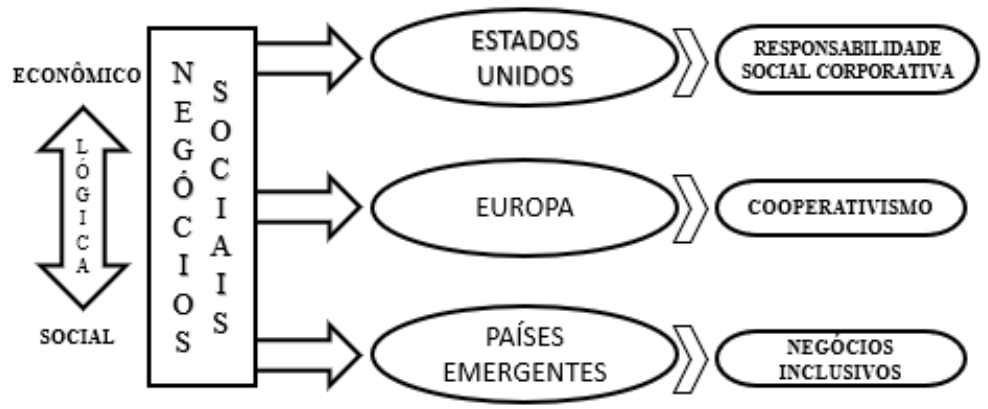


Fonte: Elaborado pelos autores (2018).

As três perspectivas dizem respeito às organizações que visam ter um impacto social positivo, especialmente a longo prazo (Comini, Barki \& Aguiar, 2012). Entretanto, existem diferenças entre as perspectivas, enquanto a perspectiva americana busca valor compartilhado, em que as organizações do setor privado usam seus pontos fortes para enfrentar os problemas sociais, a perspectiva europeia enfatiza o papel das organizações da sociedade civil e a de países emergentes diz respeito a negócios que já nascem inclusivos (Chaves et al., 2017).

A responsabilidade social corporativa (RSC) se alicerça na perspectiva norte-americana. Nesta perspectiva, as organizações que optam por ações de RSC podem ser vistas como negócios sociais e, se utilizam disto como uma estratégia para melhorar seu relacionamento com os stakeholders, manter ou aumentar sua rentabilidade e potencializar o seu desenvolvimento econômico e social (Bertoncello \& Chang Junior, 2007).

A responsabilidade social corporativa (RSC) é uma linguagem e uma perspectiva amplamente conhecida pelo mundo e vem se tornando ainda mais relevante na medida em que os diferentes stakeholders esperam que os negócios façam mais pela sociedade do que apenas fazer dinheiro e obedecer às leis. Atualmente, a ética e a filantropia ajudam a manifestar e atender as expectativas sociais que são colocadas sobre as organizações modernas (Carroll, 2015).

Não existem definições consensuais acerca da responsabilidade social corporativa (RSC). Entretanto, foi observado por Matten, Crane e Chapple (2003) que o termo teve seu desenvolvimento teórico moldado como uma temática para entender o relacionamento entre as empresas e a sociedade. Na definição de Srour (1998), a RSC deve ser entendida como uma orientação para os outros. Ela reflete tanto um sentimento de realidade quanto um olhar para o futuro.

Desde modo, a RSC pode ser entendida como uma forma das empresas se colocarem em seu contexto. Chiavenato (2002) corrobora com essa visão ao explicar que a RSC está voltada para atitude e comportamento da organização em face das exigências sociais. Depois de cumpridas as prescrições de leis e de contratos, ela constitui uma resposta da organização às necessidades da sociedade.

Já para Blasi, Caporin e Fontini (2018), a responsabilidade social corporativa pode ser amplamente definida como as atitudes positivas ou "responsáveis" de uma empresa em direção aos seus stakeholders. Essa definição está inerentemente ligada com a ideia que as firmas podem se beneficiar positivamente ao engajar com suas várias partes interessadas e públicos relevantes, tanto internos como externos.

Entretanto, foi ressaltado por Bezerra (2007) que a RSC não está relacionada ao atendimento exclusivo dos interesses dos proprietários ou acionistas, como foi o enfoque dominante até a década de 1980, nem está restrita ao atendimento das necessidades da 
comunidade - objeto específico da chamada ação social das empresas. Desta forma, ela deve ser vista como uma forma plural de gestão da corporação, que leva em consideração os interesses das várias partes envolvidas / afetadas pelo negócio.

Com relação a influência que a RSC tem sobre o desempenho financeiro (DF), existem pesquisas que apontam uma relação positiva entre RSC e DF (Rossi Júnior, 2009; Alberton \& Costa Junior, 2007). Entretanto, as evidências não têm sido apresentadas livres de questionamentos. Como resultado, a hipótese de que a RSC possui relação positiva com o DF continua inconclusiva (Cavalcanti \& Boente, 2012).

\section{Metodologia}

Esta pesquisa possui uma natureza qualitativa. A metodologia utilizada foi uma revisão sistemática da literatura internacional sobre a responsabilidade social corporativa (RSC) e o desempenho financeiro (DF). A revisão sistemática, de acordo com Galvão, Sawada e Trevizan (2004), é uma técnica útil que proporciona uma síntese organizada das informações disponíveis a respeito de um problema específico por meio do método científico. Deste modo, a revisão sistemática permite uma análise baseada nas evidências empíricas das investigações sobre o tema da pesquisa.

As fontes pesquisadas para a coleta de artigos sobre RSC e DF se deu por meio do portal de periódicos da CAPES, utilizando a conta institucional da Universidade Federal do Paraná. A partir do portal de periódicos foram acessadas duas bases de dados: Scopus e Web of Science. O período da investigação foi de 2007 a 2017.

A primeira base consultada foi o Scopus. Foram definidos os termos de busca "corporate social responsibility" e "financial performance" no título, no período de 2007 a 2017. Foi utilizado como critério de refinamento as áreas de "business, management and accounting" e "economics, econometrics and finance", chegando a 64 artigos. Deste montante, foi definido como critério de corte os trabalhos mais citados, adotando a quantia mínima de dez citações por artigo. Deste modo chegou-se ao montante total de 20 artigos para serem analisados sistematicamente.

A segunda base consultada foi o Web of Science. Nela foram definidos os termos de busca "corporate social responsibility" e "financial performance" no título, no período de 2007 a 2017. Foi utilizado como critério de refinamento as áreas de "management", "business", "business finance" e "economics", o que resultou em um total de 28 artigos. O último critério, de pesquisas com no mínimo de dez citações, resultou em um total de 7 artigos selecionados para análise.

$\mathrm{Na}$ etapa seguinte foi realizado a conferência de duplicidade dos artigos selecionados nas duas bases de dados. Se constatou 7 artigos comuns as duas bases, resultando em uma 
quantia total de 20 artigos para a análise. Seguindo a metodologia de Sarturi, Seravalli e Boaventura (2015), foram identificados e catalogados o título, ano, periódico, base de dados e autores dos trabalhos. Posteriormente foram analisados o número de citações, os objetivos, aspectos metodológicos, tamanho da amostra utilizada, país de origem do estudo e resultados dos trabalhos. A seguir, na tabela 01, foi apresentado os artigos selecionados pela revisão sistemática.

Tabela 01: Artigos Selecionados pela Revisão Sistemática.

\begin{tabular}{|c|c|c|c|c|}
\hline Ano & Título & Autores & Períodico & Base \\
\hline 2008 & $\begin{array}{c}\text { A note on the interaction between corporate } \\
\text { social responsibility and financial performance }\end{array}$ & Scholtens, B. & $\begin{array}{l}\text { Ecological } \\
\text { Economics }\end{array}$ & Scopus \\
\hline 2009 & $\begin{array}{l}\text { Corporate social responsibility and financial } \\
\text { performance: the "virtuous circle" revisited }\end{array}$ & $\begin{array}{l}\text { Nelling, E.; Webb, } \\
\text { E.; }\end{array}$ & $\begin{array}{l}\text { Review of } \\
\text { Quantitative Finance } \\
\text { and Accounting }\end{array}$ & Scopus \\
\hline 2009 & $\begin{array}{l}\text { The impact of corporate social responsibility on } \\
\text { financial performance: Evidence from business } \\
\text { in Taiwan }\end{array}$ & $\begin{array}{l}\text { Lin, C. H.; Yang, H. } \\
\text { L.; Liou, Y. }\end{array}$ & $\begin{array}{l}\text { Technology in } \\
\text { Society }\end{array}$ & Scopus \\
\hline 2010 & $\begin{array}{l}\text { Corporate social responsibility and corporate } \\
\text { financial performance: evidence from Korea }\end{array}$ & $\begin{array}{l}\text { Choi, J. S.; Kwak, } \\
\text { Y. M.; Choe, C. }\end{array}$ & $\begin{array}{c}\text { Australian Journal of } \\
\text { Management }\end{array}$ & Scopus \\
\hline 2010 & $\begin{array}{c}\text { Managing corporate performance Investigating } \\
\text { the relationship between corporate social } \\
\text { responsibility and financial performance in } \\
\text { emerging markets }\end{array}$ & $\begin{array}{l}\text { Aras, G.; Aybars, } \\
\text { A.; Kutlu, O. }\end{array}$ & $\begin{array}{l}\text { International Journal } \\
\text { of Productivity and } \\
\text { Performance } \\
\text { Management }\end{array}$ & Scopus \\
\hline 2010 & $\begin{array}{l}\text { Does it Pay to be Socially Responsible? } \mathrm{Na} \\
\text { Empirical Examination of Impact of Corporate } \\
\text { Social Responsibility on Financial Performance }\end{array}$ & $\begin{array}{l}\text { Kapoor, S.; Sandhu, } \\
\text { H. S. }\end{array}$ & $\begin{array}{l}\text { Global Business } \\
\text { Review }\end{array}$ & Scopus \\
\hline 2011 & $\begin{array}{c}\text { Effects of different dimensions of corporate } \\
\text { social responsibility on corporate financial } \\
\text { performance in tourism-related industries }\end{array}$ & Inoue, Y.; Lee, S. & $\begin{array}{c}\text { Tourism } \\
\text { Management }\end{array}$ & Scopus \\
\hline 2011 & $\begin{array}{l}\text { Corporate social responsibility, firm value and } \\
\text { financial performance in Brazil }\end{array}$ & $\begin{array}{l}\text { Crisóstomo, V. L; } \\
\text { Freire, F. S.; } \\
\text { Vasconcellos, F. C. }\end{array}$ & $\begin{array}{c}\text { SOCIAL } \\
\text { RESPONSIBILITY } \\
\text { JOURNAL } \\
\end{array}$ & Scopus \\
\hline 2011 & $\begin{array}{l}\text { Corporate social responsibility and corporate } \\
\text { financial performance in China: an empirical } \\
\text { research from Chinese firms }\end{array}$ & Chen, H.; Wang, X. & $\begin{array}{c}\text { Corporate } \\
\text { Governance: The } \\
\text { international journal } \\
\text { of business in society }\end{array}$ & Scopus \\
\hline 2011 & $\begin{array}{l}\text { Looking for evidence of the relationship between } \\
\text { corporate social responsibility and corporate } \\
\text { financial performance in an emerging market }\end{array}$ & $\begin{array}{l}\text { Saleh, M.; Zulkifli, } \\
\text { N.; Muhamad, R. }\end{array}$ & $\begin{array}{c}\text { Asia-Pacific Journal } \\
\text { of Business } \\
\text { Administration } \\
\end{array}$ & Scopus \\
\hline 2011 & $\begin{array}{l}\text { Executive Compensation, Corporate Social } \\
\text { Responsibility, and Corporate Financial } \\
\text { Performance: A Multi-Equation Framework }\end{array}$ & $\begin{array}{l}\text { Callan, S. J.; } \\
\text { Thomas, J. M. }\end{array}$ & $\begin{array}{l}\text { Corporate Social } \\
\text { Responsibility and } \\
\text { Environmental } \\
\text { Management }\end{array}$ & Scopus \\
\hline 2012 & $\begin{array}{c}\text { How Corporate Social Responsibility } \\
\text { Engagement Strategy Moderates the CSR-- } \\
\text { Financial Performance Relationship }\end{array}$ & $\begin{array}{l}\text { Tang, Z.; Hull, C. } \\
\text { E.; Rothenberg, S. }\end{array}$ & $\begin{array}{c}\text { Journal of } \\
\text { Management Studies }\end{array}$ & $\begin{array}{l}\text { Web of } \\
\text { Science }\end{array}$ \\
\hline 2012 & $\begin{array}{c}\text { Environmental corporate social responsibility } \\
\text { and financial performance: Disentangling direct } \\
\text { and indirect effects }\end{array}$ & $\begin{array}{l}\text { Lioui, A.; Sharma, } \\
\text { Z. }\end{array}$ & $\begin{array}{l}\text { Ecological } \\
\text { Economics }\end{array}$ & Scopus \\
\hline
\end{tabular}




\begin{tabular}{|c|c|c|c|c|}
\hline 2013 & $\begin{array}{l}\text { Corporate social responsibility in the banking } \\
\text { industry: Motives and financial performance }\end{array}$ & $\begin{array}{l}\text { Wua, M. W.; Shen, } \\
\text { C. H. }\end{array}$ & $\begin{array}{l}\text { Journal of Banking } \\
\text { \& Finance }\end{array}$ & Scopus \\
\hline 2013 & $\begin{array}{l}\text { The corporate social responsibility-financial } \\
\text { performance link in the U.S. restaurant industry: } \\
\text { Do economic conditions matter? }\end{array}$ & $\begin{array}{l}\text { Lee, S.; Singalb, M.; } \\
\text { Kangc, K. H. }\end{array}$ & $\begin{array}{c}\text { International Journal } \\
\text { of Hospitality } \\
\text { Management }\end{array}$ & Scopus \\
\hline 2013 & $\begin{array}{l}\text { Descriptive, instrumental and strategic } \\
\text { approaches to corporate social responsibility Do } \\
\text { they drive the financial performance of } \\
\text { companies differently? }\end{array}$ & $\begin{array}{l}\text { Boesso, G.; Kumar, } \\
\text { K.; Michelon, G. }\end{array}$ & $\begin{array}{c}\text { Accounting, } \\
\text { Auditing \& } \\
\text { Accountability } \\
\text { Journal }\end{array}$ & $\begin{array}{l}\text { Web of } \\
\text { Science }\end{array}$ \\
\hline 2014 & $\begin{array}{l}\text { How does corporate social responsibility } \\
\text { contribute to firm financial performance? The } \\
\text { mediating role of competitive advantage, } \\
\text { reputation, and customer satisfaction }\end{array}$ & $\begin{array}{l}\text { Saeidi, S. P.; Sofian, } \\
\text { S.; Saeidi, P.; Saeidi, } \\
\text { S. P.; Saaeidi, S. A. }\end{array}$ & $\begin{array}{l}\text { Journal of Business } \\
\text { Research }\end{array}$ & $\begin{array}{l}\text { Web of } \\
\text { Science }\end{array}$ \\
\hline 2014 & $\begin{array}{c}\text { Corporate Social Responsibility and Financial } \\
\text { Performance in Islamic Banks }\end{array}$ & $\begin{array}{l}\text { Farag, H.; Mallin, } \\
\text { C.; Ow-Yong, K. }\end{array}$ & $\begin{array}{c}\text { Journal of Economic } \\
\text { Behavior \& } \\
\text { Organization } \\
\end{array}$ & Scopus \\
\hline 2015 & $\begin{array}{c}\text { Does Corporate Social Responsibility Lead to } \\
\text { Superior Financial Performance? A Regression } \\
\text { Discontinuity Approach }\end{array}$ & Flammer, C. & Management Science & Scopus \\
\hline 2015 & $\begin{array}{c}\text { A Meta-Analytic Review of Corporate Social } \\
\text { Responsibility and Corporate Financial } \\
\text { Performance: The Moderating Effect of } \\
\text { Contextual Factors }\end{array}$ & $\begin{array}{l}\text { Wang, Q.; Dou, J.; } \\
\text { Jia, S. }\end{array}$ & Business \& Society & $\begin{array}{l}\text { Web of } \\
\text { Science }\end{array}$ \\
\hline
\end{tabular}

Fonte: Elaborado pelos autores com base nos trabalhos consultados.

Sequencialmente, os artigos levantados, seguem apresentados a partir de quadros e textos explicativos na próxima seção. A análise elaborada apresenta em um primeiro momento um panorama geral dos artigos investigados, em termos de evolução, regiões geográficas e indústrias onde foram elaborados, e posteriormente recai sobre a análise dos objetivos, metodologias e principais contribuições.

\section{Resultados}

Foi observado por meio da revisão sistemática que a literatura acerca da relação entre responsabilidade social corporativa (RSC) e desempenho financeiro (DF) é predominantemente quantitativa (95\%). Isso se deve ao problema de pesquisa envolver relações entre duas ou mais variáveis que se adequam melhor aos modelos de regressão e hipóteses quantitativas. Apenas $5 \%$ dos artigos analisados são qualitativos.

Embora a relação entre a RSC e o DF tenha sido amplamente explorada pela literatura, o debate ainda é contraditório e marcado por diferentes visões acerca dessa relação. Por este motivo, a literatura sobre RSC e DF ainda continua a ser explorada. A presente pesquisa selecionou os trabalhos mais relevantes no período entre 2007 e 2017. Em relação a evolução ao longo do tempo das pesquisas filtrados conforme explicitado na metodologia, a mesma pode ser observada no gráfico 01. 


\section{Gráfico 01: Evolução dos trabalhos acerca da relação entre RSC e DF.}

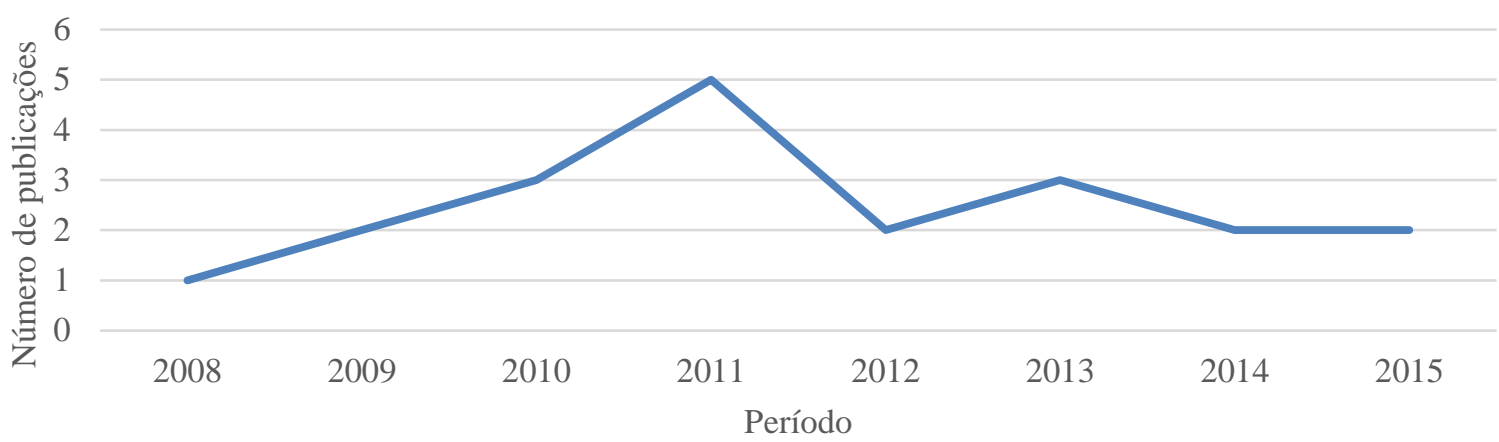

Fonte: Elaborado pelos autores com base nos trabalhos consultados.

O gráfico aponta que dos trabalhos considerados como mais relevantes conforme o critério metodológico adotado, houve um crescimento ao longo dos anos que partiu do menor valor observado de 1 artigo em 2008 e atingiu seu maior valor de 5 artigos no ano de 2011. Em termos de média, foram selecionados dois artigos por ano para compor a amostra. Cabe ressaltar que pelo critério estabelecido, não foram observadas pesquisas nos anos de 2007, 2016 e 2017.

Os filtros aplicados sobre as bases de dados permitiram capturar uma grande heterogeneidade de regiões geográficas (gráfico 02) em uma variedade de indústrias (gráfico 03). A revisão sistêmica capturou pesquisas conduzidas em pelo menos quatro continentes, entre eles a Ásia foi predominante, (6 pesquisas); seguido pela América do Norte (3 pesquisas); América do Sul (1 pesquisa); Europa (1 trabalho). Os países onde as pesquisas foram realizadas podem ser observados no gráfico 02 .

\section{Gráfico 02: Países em que as pesquisas foram realizadas.}

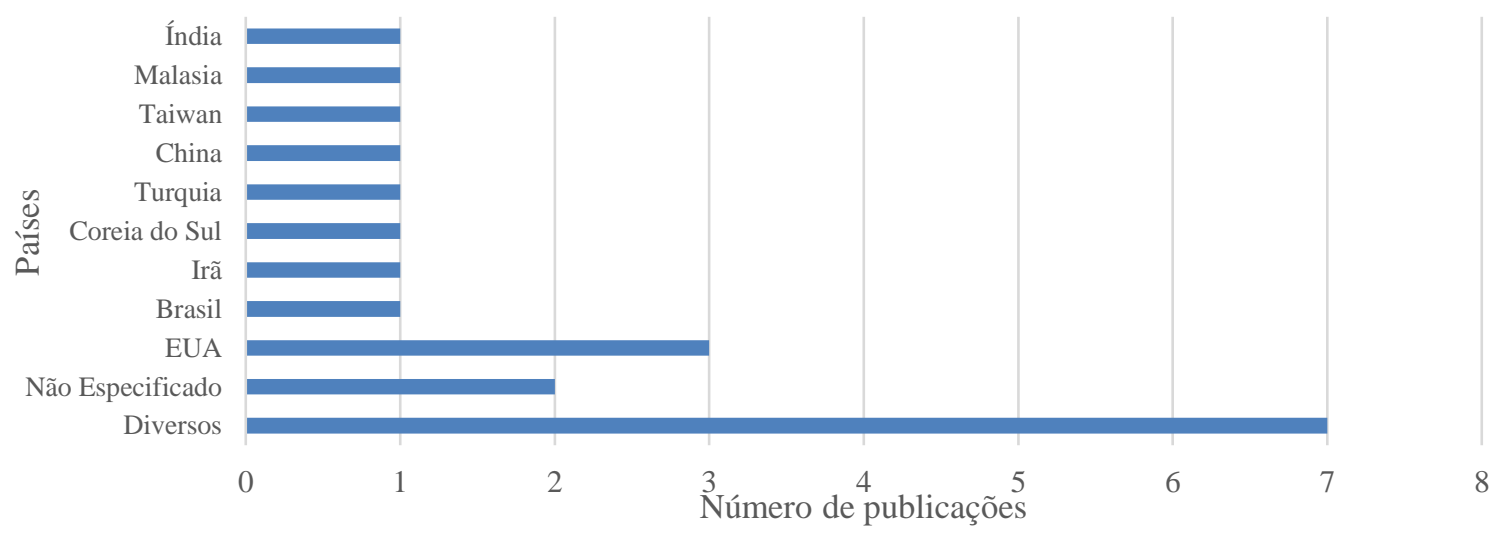

Fonte: Elaborado pelos autores com base nos trabalhos consultados.

Se observa que a maior parte dos artigos (7 pesquisas) foram realizadas em diversos países. A maioria destes utilizou amostras a partir de índices de diversas bolsas de valores e de 
bases de dados internacionais, o que dificultou a identificação precisa dos países em que essas pesquisas empíricas ocorreram. Em dois artigos não foi possível identificar onde os autores realizaram a pesquisa. Dos nove artigos que foram conduzidos em apenas um país, apenas os EUA obtiveram mais de uma observação (Scholtens, 2008; Nelling \& Webb, 2009; Lee, Singalb \& Kangc, 2013). Já em relação aos setores onde a pesquisa foi realizada, os mesmos podem ser observados no gráfico 03 .

\section{Gráfico 03: Indústrias em que as pesquisas foram realizadas.}

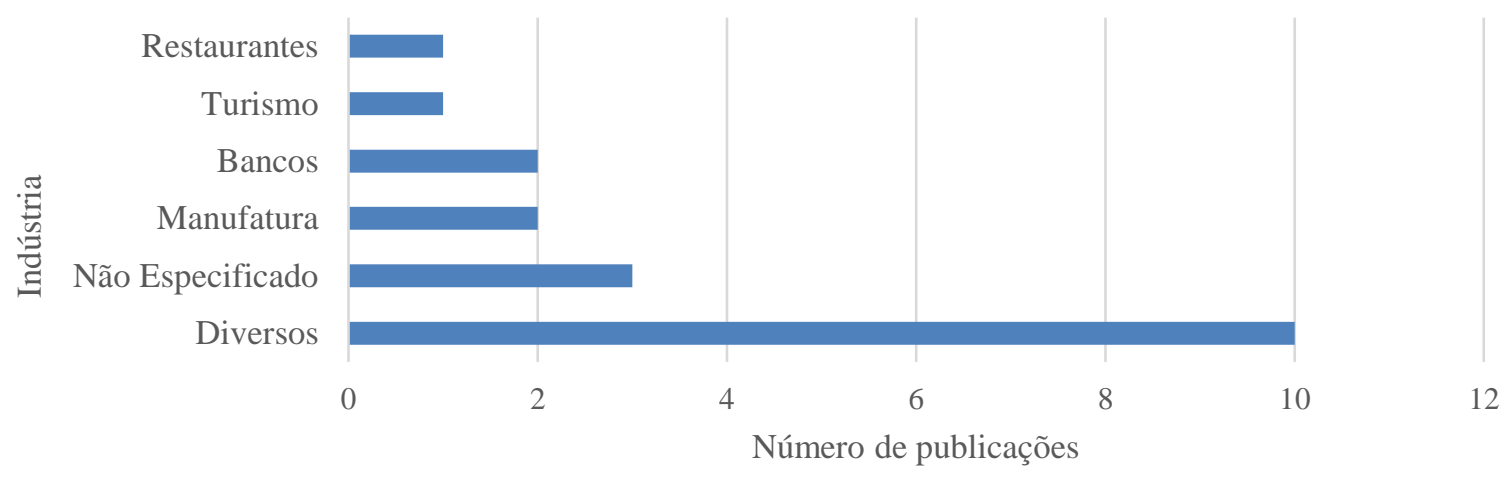

Fonte: Elaborado pelos autores com base nos trabalhos consultados.

As indústrias contempladas nos trabalhos selecionados incluem o setor bancário (Wua \& Shen, 2013; Farag, Mallin \& Ow-Yong, 2014), o setor de manufatura (Lin, Yang \& Liou, 2009; Saeidi et al., 2014), restaurantes (Lee, Singalb \& Kangc, 2013), turismo (Inoue \& Lee, 2011) além das outras 12 pesquisas que não deram ênfase em setores específicos, abrangendo diversas indústrias.

Conforme apontado anteriormente, a literatura acerca da RSC e sua relação com o DF ainda é contraditória. Para tanto, foi realizado um levantamento com o objetivo e as principais contribuições de cada artigo, considerando seus aspectos metodológicos, de forma a se verificar o estado da arte do link entre os dois conceitos. Os objetivos e os resultados dos artigos selecionados podem ser observados no quadro 02 .

Quadro 02: Objetivos e resultados dos artigos selecionados.

\begin{tabular}{|l|l|l|}
\hline Artigo & Objetivo & Principais Contribuições \\
\hline $\begin{array}{l}\text { Aras, Aybars } \\
\text { e Kutlu } \\
(2010) .\end{array}$ & $\begin{array}{l}\text { Este artigo tem como objetivo investigar } \\
\text { a relação entre responsabilidade social } \\
\text { corporativa (RSC) e o desempenho } \\
\text { financeiro (DF) da firma. }\end{array}$ & $\begin{array}{l}\text { Os autores encontraram uma relação entre o tamanho da } \\
\text { empresa a RSC. No entanto, os autores não } \\
\text { conseguiram encontrar uma relação significativa entre } \\
\text { RSC e DF. }\end{array}$ \\
\hline $\begin{array}{l}\text { Boesso, } \\
\text { Kumar e }\end{array}$ & $\begin{array}{l}\text { Os autores investigam se os aspectos } \\
\text { descritivos, instrumentais e }\end{array}$ & $\begin{array}{l}\text { Foi evidenciado que as abordagens da RSC - descritiva, } \\
\text { instrumental e estratégica - estão associadas ao DF de } \\
\text { (2013). }\end{array}$ \\
$\begin{array}{l}\text { abordagens estratégicas para a RSC estão } \\
\text { delacionadas ao DF, bem como a natureza } \\
\text { desse relacionamento, se houver. }\end{array}$ & tem uma associação positiva com DF de curto prazo; a \\
\hline
\end{tabular}




\begin{tabular}{|c|c|c|}
\hline & & $\begin{array}{l}\text { estratégica está associada ao DF de curto e médio prazo; } \\
\text { e a descritiva não tem associação com o DF. }\end{array}$ \\
\hline $\begin{array}{l}\text { Callan, Janet e } \\
\text { Thomas } \\
\text { (2011). }\end{array}$ & $\begin{array}{l}\text { O objetivo é identificar e medir os } \\
\text { determinantes e as relações entre } \\
\text { remuneração de executivos, DF e RSC. }\end{array}$ & $\begin{array}{l}\text { Entre os resultados, a suposição de endogeneidade é } \\
\text { suportada, o que significa que o DF e o desempenho } \\
\text { social são determinados simultaneamente. }\end{array}$ \\
\hline $\begin{array}{l}\text { Chen e Wang } \\
(2011) \text {. }\end{array}$ & $\begin{array}{l}\text { Este artigo visa utilizar a teoria dos } \\
\text { stakeholders para responder se a RSC tem } \\
\text { relação com o DF em empresas chinesas. }\end{array}$ & $\begin{array}{l}\text { Os resultados mostram que a RSC pode melhorar o DF } \\
\text { do ano atual, e ter efeitos significativos no próximo ano, } \\
\text { e vice-versa. }\end{array}$ \\
\hline $\begin{array}{l}\text { Choi, Kwak e } \\
\text { Choe (2010). }\end{array}$ & $\begin{array}{l}\text { Este artigo estuda a relação empírica entre } \\
\text { responsabilidade social corporativa } \\
\text { (RSC) e desempenho financeiro } \\
\text { corporativo na Coréia. }\end{array}$ & $\begin{array}{l}\text { Os autores encontraram uma relação positiva e } \\
\text { significativa e robusta entre o desempenho financeiro } \\
\text { das empresas e o índice de RSC ponderado pelos } \\
\text { stakeholders. }\end{array}$ \\
\hline $\begin{array}{l}\text { Crisóstomo, } \\
\text { Freire e } \\
\text { Vasconcellos } \\
\text { (2011). }\end{array}$ & $\begin{array}{l}\text { O objetivo do trabalho é examinar a } \\
\text { relação entre RSC e DF, considerando o } \\
\text { valor da empresa e o desempenho } \\
\text { contábil financeiro, em um mercado } \\
\text { emergente - o Brasil. }\end{array}$ & $\begin{array}{l}\text { Os resultados indicam que a RSC está destruindo o valor } \\
\text { no Brasil, uma vez que foi encontrada uma correlação } \\
\text { negativa significativa entre o RSC e o valor da empresa. }\end{array}$ \\
\hline $\begin{array}{l}\text { Farag, Mallin } \\
\text { e Yong } \\
(2014) .\end{array}$ & $\begin{array}{l}\text { O objetivo deste trabalho é examinar a } \\
\text { relação entre a RSC e o desempenho } \\
\text { financeiro em bancos islâmicos }\end{array}$ & $\begin{array}{l}\text { A análise empírica destaca uma associação positiva } \\
\text { entre a divulgação da RSC e o desempenho financeiro. }\end{array}$ \\
\hline $\begin{array}{l}\text { Flammer } \\
(2015) .\end{array}$ & $\begin{array}{l}\text { Este estudo examina o efeito das } \\
\text { propostas de acionistas relacionadas à } \\
\text { RSC no DF. Com ênfase em propostas de } \\
\text { RSC que passam ou falham por uma } \\
\text { pequena margem de votos. }\end{array}$ & $\begin{array}{l}\text { A adoção de propostas de RSC leva a DF superior, o que } \\
\text { implica que essas propostas são valorizantes. A } \\
\text { produtividade e as vendas aumentam após a votação da } \\
\text { RSC. Entretanto, o autor salienta que os resultados não } \\
\text { são generalizáveis. }\end{array}$ \\
\hline $\begin{array}{l}\text { Inoue e Lee } \\
(2011) .\end{array}$ & $\begin{array}{l}\text { O objetivo deste estudo foi desagregar a } \\
\text { RSC em cinco dimensões principais de } \\
\text { stakeholders: (1) relações com } \\
\text { funcionários, (2) qualidade do produto, } \\
\text { (3) relações comunitárias, (4) questões } \\
\text { ambientais e (5) diversidade e examinar } \\
\text { como cada dimensão impacta o DF. }\end{array}$ & $\begin{array}{l}\text { os resultados revelaram que cada dimensão tem um } \\
\text { efeito diferente no DF a curto prazo e no futuro. Os } \\
\text { resultados variaram em cada indústria. As descobertas } \\
\text { podem fornecer aos gestores informações sobre quais as } \\
\text { dimensões das atividades de RSC que melhorariam o } \\
\text { DF. }\end{array}$ \\
\hline $\begin{array}{l}\text { Kapoor e } \\
\text { Sandhu } \\
(2010) \text {. }\end{array}$ & $\begin{array}{l}\text { O artigo tenta examinar o impacto da } \\
\text { RSC no DF em termos de rentabilidade e } \\
\text { crescimento após o controle do efeito de } \\
\text { outras variáveis no desempenho } \\
\text { financeiro. }\end{array}$ & $\begin{array}{l}\text { Existe um impacto positivo e significativo da RSC no } \\
\text { DF e no crescimento corporativo. Deste modo é útil para } \\
\text { os gerentes considerar o impacto positivo da RSC no DF } \\
\text { ao tomar decisões sobre investir em RSC. }\end{array}$ \\
\hline $\begin{array}{l}\text { Lee, Singalb e } \\
\text { Kangc (2013). }\end{array}$ & $\begin{array}{l}\text { Este estudo examina } \text { o papel } \\
\text { desempenhado pelas condições } \\
\text { econômicas (períodos de recessão) no } \\
\text { vínculo entre RSC e DF nos EUA. }\end{array}$ & $\begin{array}{l}\text { Os resultados mostram que a RSC não teve um impacto } \\
\text { significativo no DF, mesmo com a inclusão de } \\
\text { condições econômicas. Este resultado sugere que o } \\
\text { principal efeito do RSC no DF não é significativo, } \\
\text { independentemente das diferentes dimensões RSC. }\end{array}$ \\
\hline $\begin{array}{l}\text { Lin, Yang e } \\
\text { Liou (2009). }\end{array}$ & $\begin{array}{l}\text { Os autores tentam preencher a lacuna ao } \\
\text { analisar e integrar a teoria em relação à } \\
\text { RSC e o DF, sugerindo que essa relação } \\
\text { provavelmente é mediado por uma série } \\
\text { de variáveis-chave. }\end{array}$ & $\begin{array}{l}\text { Com base em asserções teóricas e evidências empíricas } \\
\text { na literatura, os autores identificam uma relação positiva } \\
\text { entre RSC e DF. Embora o RSC tenha pouco impacto } \\
\text { positivo no DF de curto prazo, ele oferece uma notável } \\
\text { vantagem fiscal a longo prazo. }\end{array}$ \\
\hline $\begin{array}{l}\text { Lioui e } \\
\text { Sharma } \\
(2012)\end{array}$ & $\begin{array}{l}\text { Este artigo avalia o impacto da } \\
\text { responsabilidade social corporativa } \\
\text { ambiental (RSCA) no DF, medido pelo } \\
\text { ROA e pela Q. de Tobin. }\end{array}$ & $\begin{array}{l}\text { As evidências apontam que a relação entre o retorno } \\
\text { sobre ativos (ROA) e a RSCA das empresas, é negativa } \\
\text { e estatisticamente significante. }\end{array}$ \\
\hline
\end{tabular}




\begin{tabular}{|c|c|c|}
\hline $\begin{array}{l}\text { Nelling e } \\
\text { Webb (2009). }\end{array}$ & $\begin{array}{l}\text { Os autores examinam a relação causal } \\
\text { entre responsabilidade social corporativa } \\
\text { (RSC) e o desempenho financeiro. }\end{array}$ & $\begin{array}{l}\text { Os resultados sugerem que o desempenho no mercado } \\
\text { de ações leva para um maior investimento em RSC. } \\
\text { Entretanto, a RSC não afeta o desempenho financeiro. }\end{array}$ \\
\hline $\begin{array}{l}\text { Saeidi et al. } \\
(2014) \text {. }\end{array}$ & $\begin{array}{l}\text { Este estudo investiga a vantagem } \\
\text { competitiva sustentável, reputação e } \\
\text { satisfação do cliente como três } \\
\text { mediadores prováveis na relação entre } \\
\text { RSC e DF. }\end{array}$ & $\begin{array}{l}\text { O efeito positivo da RSC no DF deve-se ao efeito } \\
\text { positivo que a RSC tem na vantagem competitiva, } \\
\text { reputação e satisfação do cliente. Porém apenas a } \\
\text { reputação e a vantagem competitiva medeiam a relação } \\
\text { entre a RSC e o DF. }\end{array}$ \\
\hline $\begin{array}{l}\text { Saleh, Zulkifli } \\
\text { e Muhamad } \\
\text { (2011). }\end{array}$ & $\begin{array}{l}\text { O objetivo deste trabalho é examinar a } \\
\text { relação entre RSC e o DF das empresas de } \\
\text { capital aberto da Malásia (PLCs), } \\
\text { caracterizada como um mercado } \\
\text { emergente. }\end{array}$ & $\begin{array}{l}\text { Os resultados indicam que há relações positivas e } \\
\text { significativas da RSC no DF. Duas das dimensões da } \\
\text { RSC, nomeadamente as relações com os funcionários e } \\
\text { o envolvimento da comunidade, foram positivamente } \\
\text { relacionadas ao DF. Entretanto há evidências limitadas } \\
\text { dessa relação no longo prazo. }\end{array}$ \\
\hline $\begin{array}{l}\text { Scholtens } \\
\text { (2008). }\end{array}$ & $\begin{array}{l}\text { O objetivo é estudar a interação entre o } \\
\text { desempenho social e o financeiro, } \\
\text { considerando tanto aspectos sociais como } \\
\text { de envolvimento na comunidade, nas } \\
\text { relações com funcionários, diversidade e } \\
\text { meio ambiente. }\end{array}$ & $\begin{array}{l}\text { As evidências apontam que a direção da "causação" } \\
\text { corre predominantemente do DF para o social. No } \\
\text { entanto, os padrões de interação específicos tendem a } \\
\text { variar ao longo das diferentes dimensões. }\end{array}$ \\
\hline $\begin{array}{l}\text { Tang, Hull e } \\
\text { Rothenberg } \\
\text { (2012). }\end{array}$ & $\begin{array}{l}\text { Os autores propõem que os lucros das } \\
\text { firmas são moldados por como as } \\
\text { empresas engajam na RSC e propõem } \\
\text { quatro hipóteses para serem testadas. }\end{array}$ & $\begin{array}{l}\text { Os dados apontam que as empresas se beneficiam mais } \\
\text { ao adotar estratégias consistentes de envolvimento na } \\
\text { RSC, e começam com aspectos que são mais internos à } \\
\text { empresa. No entanto, o ritmo do envolvimento na RSC, } \\
\text { não modera o relacionamento RSC-DF. }\end{array}$ \\
\hline $\begin{array}{l}\text { Wang, Dou e } \\
\text { Jia }(2015) .\end{array}$ & $\begin{array}{l}\mathrm{O} \text { artigo tem como objetivo revisar } \\
\text { sistematicamente a literatura e quantificar } \\
\text { o link entre a RSC e o DF por meio de } \\
\text { uma meta-análise. }\end{array}$ & $\begin{array}{l}\text { Os autores estimam os efeitos do relacionamento entre a } \\
\text { RSC e o DF e evidenciam uma relação positiva e } \\
\text { significante, ressaltando o argumento de que a RSC } \\
\text { aprimora o DF. }\end{array}$ \\
\hline $\begin{array}{l}\text { Wu e Shen } \\
\text { (2013). }\end{array}$ & $\begin{array}{l}\text { Este estudo investiga a associação entre } \\
\text { RSC e DF, e discute os motivos que } \\
\text { levam os bancos a se envolverem com } \\
\text { RSC. }\end{array}$ & $\begin{array}{l}\text { As evidências empíricas mostram que a RSC se associa } \\
\text { positivamente ao DF em termos de retorno sobre ativos, } \\
\text { retorno sobre o patrimônio líquido, renda líquida de } \\
\text { juros e renda sem juros. }\end{array}$ \\
\hline
\end{tabular}

Fonte: Elaborado pelos autores com base nos trabalhos consultados.

Os artigos selecionados para verificar a relação entre a RSC e o DF possuem objetivos e metodologias muito semelhantes em função da especificidade do tema. De uma forma geral, 19 os artigos visam explicitar a relação quantitativa entre os dois conceitos, e variam em termos das variáveis selecionadas para mediar essa relação. Apenas o trabalho de Wang, Dou e Jia (2015) utilizou a metodologia qualitativa elaborando uma meta-análise de trabalhos anteriores.

Os estudos exploram diferentes dimensões da RSC. O trabalho de Scholtens (2008) que inclui elementos de meio ambiente e diversidade e envolvimento na comunidade; Lioui e Sharma (2012) investigam a responsabilidade social corporativa ambiental (RSCA); Inoue e Lee (2011) utilizam cinco dimensões incluindo os funcionários, produto, comunidade, meio ambiente e diversidade.

As pesquisas analisadas também tiveram seus objetivos variados em termos das variáveis e mediadores utilizados para a RSC. A investigação de Saeidi et al. (2014) utilizou a

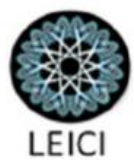


vantagem competitiva sustentável, reputação e satisfação do cliente como mediadores na relação entre RSC-DF; Lin, Yang e Liou (2009) utilizaram uma série de variáveis, como investimentos em P\&D; Callan, Janet e Thomas (2011) acrescentam a remuneração dos executivos na relação; e Boesso, Kumar e Michelon (2013) investigam aspectos descritivos, instrumentais e abordagens estratégicas.

Enquanto maior parte dos trabalhos tem por objetivo evidenciar a relação entre os conceitos, alguns trabalhos investigaram mais a fundo outros fatores. Entre esses, Wu e Shen, (2013) investigaram os motivos que levam os bancos a se envolver com a RSC; Lee, Singalb e Kangc (2013) buscam entender quais os efeitos da recessão econômica no relacionamento entre RSC e DF; e Flammer (2015) que examina os efeitos das propostas de acionistas em relação à RSC e DF.

Em termos das principais contribuições e os resultados obtidos nas vinte pesquisas, se observou que os mesmos carecem de um consenso. Entretanto, $75 \%$ dos artigos evidenciaram uma relação positiva entre a RSC e o DF. 50\% dos artigos analisados encontraram uma relação positiva direta, esses trabalhos apontam para resultados significantes de que a RSC impacta no DF. $25 \%$ das pesquisas apontam para uma relação positiva indireta entre a RSC e o DF, esses trabalhos não encontraram relações significantes ou os mecanismos pelos quais isso ocorre são desconhecidos. Os outros $25 \%$ dos artigos não encontraram evidências que existe uma relação positiva, ou evidenciaram que a RSC destrói o valor para a organização.

Entre os trabalhos que encontraram uma relação positiva, foi evidenciado por Saleh, Zulkifli e Muhamad (2011), Inoue e Lee (2011), Chen e Wang (2011) e Boesso, Kumar e Michelon (2013) que enquanto a RSC influência no DF a curto prazo, não foram encontradas evidências de que a RSC impacta no DF de longo prazo. Em contraponto, a pesquisa de Lin, Yang e Liou (2009) apontam que enquanto a RSC tem pouco impacto no DF de curto prazo, ela oferece vantagem fiscal no longo prazo.

Alguns trabalhos encontraram uma relação positiva indireta, como Saeidi et al. (2014) que evidenciaram que a RSC influência no DF por meio da reputação e da vantagem competitiva; Flammer (2015) aponta que após a votação da RSC com os acionistas, a produtividade e as vendas tendem a aumentar; Lin, Yang e Liou (2009) utilizaram os investimentos em P\&D como variável crítica da relação; Boesso, Kumar e Michelon, (2013) encontraram que as diferentes abordagens se associam de forma diferente com o DF; e Scholtens (2008), encontrou a relação inversa, é o DF que influência na melhoria da RSC.

Entre as pesquisas que encontraram relação negativa entre a RSC e o DF, Nelling e Webb (2009) evidenciam que o desempenho no mercado de ações leva a um maior investimento em RSC, entretanto os autores não encontraram relação entre RSC e DF; Lee, Singalb e Kangc (2013) e Aras, Aybars e Kutlu (2010) também não encontraram relação positiva; Lioui e Sharma (2012) encontraram uma relação negativa e estatisticamente significante; e a pesquisa

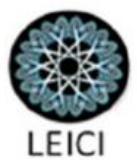


de Crisóstomo, Freire e Vasconcellos (2011) aponta que a RSC está destruindo valor já que foi encontrada uma relação negativa e significativa entre RSC e o valor da empresa.

\section{Conclusão}

Os resultados obtidos evidenciam que a relação entre a responsabilidade social corporativa (RSC) e o desempenho financeiro (DF) ainda é fragmentada na literatura. Os diferentes artigos analisados examinam várias abordagens e formas de entender a RSC, bem como inúmeras variáveis que podem influenciar na relação entre os dois conceitos.

De uma forma geral, as contribuições de $75 \%$ dos artigos analisados suportam que a RSC tem um impacto positivo no DF contra $25 \%$ das pesquisas que não encontraram ou contrariam tal relação. Entretanto cabe ressaltar que os resultados não são generalizáveis, e a RSC tende a ter um resultado positivo no DF desde que seja guiada por uma estratégia corporativa coerente. Adicionalmente a isso, existem especificidades geográficas e de indústrias que podem influenciar.

Todos os artigos analisados têm por objetivo verificar a relação entre a RSC e o DF. Consequentemente a isso, foi observado uma homogeneidade em termos metodológicos, pois a maioria dos trabalhos se utilizam de modelos de regressão e instrumentos estatísticos tradicionais para verificar a relação entre a RSC e o DF.

A falta de uma pluralidade de metodologias pode ser considerada uma limitação da literatura acerca da RSC e o DF. Essa observação foi constatada a partir do trabalho de Nelling e Webb (2009). Os autores constataram que a RSC parece ter relação com o DF quando se utilizam métodos estatísticos tradicionais. Entretanto, quando os autores utilizaram uma série temporal ajustada eles descobriram que essa relação entre RSC e DF é muito mais fraca do que anteriormente se pensava.

Uma das contribuições da presente pesquisa é chamar atenção acerca da lacuna de trabalhos com metodologias diferentes dos modelos de regressão e métodos estatísticos tradicionais. Esses métodos têm se mostrado ineficientes em dar respostas concretas, o que é evidenciado pela vasta literatura a respeito da relação entre a RSC e o DF. Atenta-se também as contribuições de Nelling e Webb (2009), que obtiveram resultados diferentes e significantes ao adotar outro procedimento metodológico.

Deste modo, em relação as pesquisas futuras, se atenta acerca da importância de pesquisas com diferentes ênfases metodológicas do que as que vem sendo tradicionalmente adotadas. É de destacada importância também pesquisas que tem por objetivo ir além de simplesmente estabelecer relações entre os dois conceitos, mas que englobem aspectos estratégicos e explicativos, pois se evidenciou que as pesquisas buscam responder se há a relação, mas falham em explicar o como isso ocorre. 
Entre as limitações da presente pesquisa, se destaca a pequena amostra selecionada dos artigos em relação a vasta literatura sobre o tema. O presente trabalho buscou capturar os trabalhos mais relevantes e com maior quantidade de citações, mas ao faze-lo deixou de lado artigos que poderiam oferecer importantes contribuições.

\section{Referencias}

Alberton, A.; Costa Junior, N. C. A. (2007). Meio ambiente e desempenho econômicofinanceiro: benefícios dos sistemas de gestão ambiental (SGAs) e o impacto da ISO 14001 nas empresas brasileiras. RAC-Eletrônica, Rio de Janeiro, v. 1, n. 2, p. 153-171.

Azevedo, A. C; Santos, D. P; Boaventura, J. M. G. (2016). Corporate social performance: Análise da evolução do construto nos estudos empíricos publicados entre 1975 e 2014. Revista Perspectivas Contemporâneas, v. 11, n. 3, p. 19-38, set./dez.

Baraibar-Diez, E.; Sotorrío, L. L. (2018). O efeito mediador da transparência na relação entre responsabilidade social corporativa e reputação corporativa. Revista Brasileira de Gestão e Negócios, São Paulo, v.20 n.1, p. 5-21, jan-mar.

Barakat, S. R; Boaventura, J. M. G; Polo, E. F. (2017). Alinhamento estratégico da responsabilidade social corporativa: um estudo de caso no setor bancário brasileiro. REAd | Porto Alegre - Edição 86 - N 1 - Janeiro / Abril - p. 206 - 233

Barki, Edgard. (2015). Negócios de Impacto: Tendência ou Modismo? GV Executivo, v. 14, p. 14-17.

Battilana, Julie; Dorado, Silvia. (2010). Building sustainable hybrid organizations: The case of commercial microfinance organizations. Academy of Management Journal, v. 53, n. 6, p. 14191440 .

Battilana, Julie; Lee, Matthew. (2014). Advancing research on hybrid organizing-Insights from the study of social enterprises. The Academy of Management Annals, v. 8, n. 1, p. 397-441.

Bertoncello S. L. T; Chang Junior, J. (2007). A importância da Responsabilidade Social Corporativa como fator de diferenciação. Revista da Faculdade de Comunicação FAAP (FACOM), 17, 70-76.

Bezerra, R. B. (2007). Responsabilidade Social Corporativa: Uma Proposta Metodológica para Orientação De Iniciativas. Dissertação de mestrado, Universidade Federal do Rio de Janeiro.

Blasi, S.; Caporin, M.; Fontini, F. (2018). A Multidimensional Analysis of the Relationship Between Corporate Social Responsibility and Firms' Economic Performance. Ecological Economics, 147, 218-229.

Carroll, A. B. (2015). Corporate social responsibility: The centerpiece of competing and complementary frameworks. Organizational Dynamics, 44, 87-96. 
Cavalcanti, J. M. M.; Boente, D. R. (2012). A relação de risco e retorno nas empresas integrantes do Índice de Sustentabilidade Empresarial no período de 2008 a 2010. Revista Ambiente Contábil, Natal, v. 4, n. 1, p. 51-71.

Chaves, T.J; Rocha, T. V; Reuther, J; Galhanone, R. F. (2017). Social business in multinational corporations: an analysis of marketing practices. São Paulo, v.12. n. 1, p. 62-75, jan/abr.

Chiavenato, I. (2002). Recursos Humanos. Edição compactada. São Paulo: Editora Atlas. $7^{\text {a }}$ Edição.

Cochran, P. F.; Wood, R. A. (1984). Corporate Social Responsibility and Financial Performance. The Academy of Management Journal, Vol. 27, No. 1, pp. 42-56.

Comini, G. M; Barki, E; Aguiar, L. T. (2012). A three-pronged approach to social business: a Brazilian multi-case analysis. Revista de Administração (FEA-USP), v. 47, p. 385-397.

Galvão, C. M.; Sawada, N. O.; Trevizan, M. A. (2004). Revisão Sistemática: Recurso que Proporciona a Incorporação das Evidências na Prática da Enfermagem. Revista LatinoAmericana de Enfermagem, v. 12, n. 3, p. 549-556.

Hull, C. E.; Rothenberg, S. (2008). Firm Performance: The Interactions of Corporate Social Performance with Innovation and Industry Differentiation. Strategic Management Journal, 29: 781-789.

Jamali, D.; Karam, C. (2016). Corporate Social Responsibility in Developing Countries as an Emerging Field of Study. International Journal of Management Reviews, Vol. 00, 1-30.

Kim, K. H; Kim, M.; Qian, C. (2015). Effects of Corporate Social Responsibility on Corporate Financial Performance: A Competitive-Action Perspective. Journal of Management, vol. XX No. X, Month XXXX 1-22.

Li, J. J.; Zhou, K. Z.; Shao, A. T. (2009). Competitive position, managerial ties, and profitability of foreign firms in China: An interactive perspective. Journal of International Business Studies, 40: 339-352.

Marques, N. S; Faria, A. M; Sbragia, R; Oliveira Junior, M. M. (2016). Incubadoras de empresas: Análise bibliométrico da produção cientifica entre 1985 e 2014. Revista Livre de Sustentabilidade e Empreendedorismo, v. 1, n. 3, p. 49-70, set-out.

Matten, D.; Crane, A.; Chapple, W. (2003). Behind the Mask: Revealing the True Face of Corporate Citizenship. Journal of Business Ethics. Vol. 45, p.109-120.

Prahalad, C. K. (2005). The Fortune at the Botton of the Pyramid: Eradicating Poverty through Profits, Upper Saddle River, NJ: Wharton School Publishing.

Prahalad, C. K.; Hart, Stuart. (2002). The Fortune at the Bottom of the Pyramid. Strategy +Business, Chicago, v. 22, p. 1-14, Jan.

Ribeiro, H. C. M; Tavares, V. C. M; Costa, B. K. (2016). Cocriação de valor: uma bibliometria de 2000 a 2014. Revista Eletrônica de Estratégia \& Negócios, Florianópolis, v.9, n.1, jan./abr. 
Rossi Júnior, J. L. (2009). What is the value of corporate social responsibility? An answer from Brazilian sustainability index. Journal of International Business and Economics, Madison, v. 9, p. $169-178$.

Sarturi, G; Seravalli, C; Boaventura, J. M. G. (2015). Afinal o que é distribuir valor para os stakeholders? Uma análise bibliográfica sobre o tema. Rev. Adm. UFSM, Santa Maria, v. 8, Ed. Especial XVI ENGEMA, p. 92-113.

Srour, R. H. (1998). Poder, cultura e ética nas organizações. Rio de Janeiro: Editora Campus. $8^{\mathrm{a}}$ Edição.

Teodósio, A. S. S; Comini, G. M. (2012). Inclusive business and poverty: prospects in the Brazilian context. Revista de Administração (FEA-USP), v. 47, p. 410-421.

Thornton, P. H; Ocasio, W. (2008). Institutional Logics. In: GREENWOOD, R.; OLIVER, C.; SAHLIN, K.; SUDDABY, R. The Sage Handbook of Organizational Institutionalism. Great Britain: Sage Publications, p. 99-129.

Waddock, S. A.; Graves, S. B. (1997). The Corporate Social Performance- Financial Performance Link. Strategic Management Journal, Vol. 18:4, 303-319. 